\title{
Der glückliche Landarzt
}

François Burnier

Korrespondenz:

Dr. med. François Burnier 8 , rue de l'auberge CH-1142 Pampigny

francois.burnier@gmx.ch
Allein auf weiter Flur, fern von aller Kultur, ständig auf Draht und im Notfalldienst, zu jeder Stunde von Patienten belästigt, die nicht unbedingt krank sind und ungehindert direkt an der Türe anklopfen ...

Das Bild des isolierten, erschöpften und der Depression ausgelieferten Landarztes scheint bei der Bevölkerung noch stark verbreitet, namentlich bei den Stadtärzten. Tatsächlich arbeiten gewisse Kollegen in Berggegenden oft unter besonders schwierigen Verhältnissen. Dies ist aber nicht immer der Fall, wie ich darzulegen versuche - ich möchte jede Verallgemeinerung meiden und mich einzig auf meine persönliche Erfahrung stützen.

Ich hatte gleichzeitig beschlossen, Medizin zu studieren und Allgemeinpraktiker zu werden. Es war 1963, und ich glaube, dass ich als Einziger meines Jahrganges schon von Anfang an diese Entscheidung getroffen hatte. «Du bist verrückt», sagten die einen. «Hast du keine höheren Ambitionen?», fragten mich andere. (Doch: Ich hatte den Ehrgeiz, gute Allgemeinmedizin zu betreiben, und dies als Landarzt. Übrigens, schon mein Vater war ein glücklicher Allgemeinarzt.)

Während meines Jahres als Chirurgieassistent am Krankenhaus Aigle begegnete ich einer Kollegin aus Basel, die Assistentin auf der chirurgischen Abteilung in Sitten war und sich später auch der Allgemeinmedizin widmen wollte. Wir haben 1973 geheiratet.
Die Hälfte unserer Assistenzjahre haben wir in der Romandie verbracht, die andere in Basel, wo unsere Tochter geboren wurde, die inzwischen auch Ärztin geworden ist. Zwei Jahre später kam unser Sohn in der Geburtsabteilung des damaligen Bezirksspitals St-Loup zur Welt.

\section{Eine ideale Gegend}

Auf dem Land ist der Allgemeinpraktiker am rechten Platz, denn da kann er seine erlernten Kenntnisse zur Anwendung bringen, nicht nur in Innerer Medizin, sondern auch in Kleinchirurgie, Gynäkologie, Pädiatrie, ORL, Dermatologie und anderen Spezialitäten. Er wird in der Regel als erster aufgesucht und hat so die Gelegenheit, die Patienten «im Urzustand» zu sehen. Nie werde ich jenen Hausbesuch für eine angebliche Grippe vergessen: Was gibt es Banaleres als eine Grippe? Der Patient aber zeigte Symptome, die mich an einen Botulismus denken liessen, eine Diagnose, die letzen Endes bestätigt wurde.

Ich liebe die Vielfalt der Patienten und die Probleme, die es zu lösen gilt. Dies erweist sich auch als besonders interessant, will man dem Patienten in seiner Ganzheit und in seinem sozialen Umfeld begegnen. Sowohl auf dem Land als in der Stadt spielt der Hausarzt eine wichtige Rolle bei der Betreuung von Patienten mit Familiensorgen, Arbeitsproblemen,

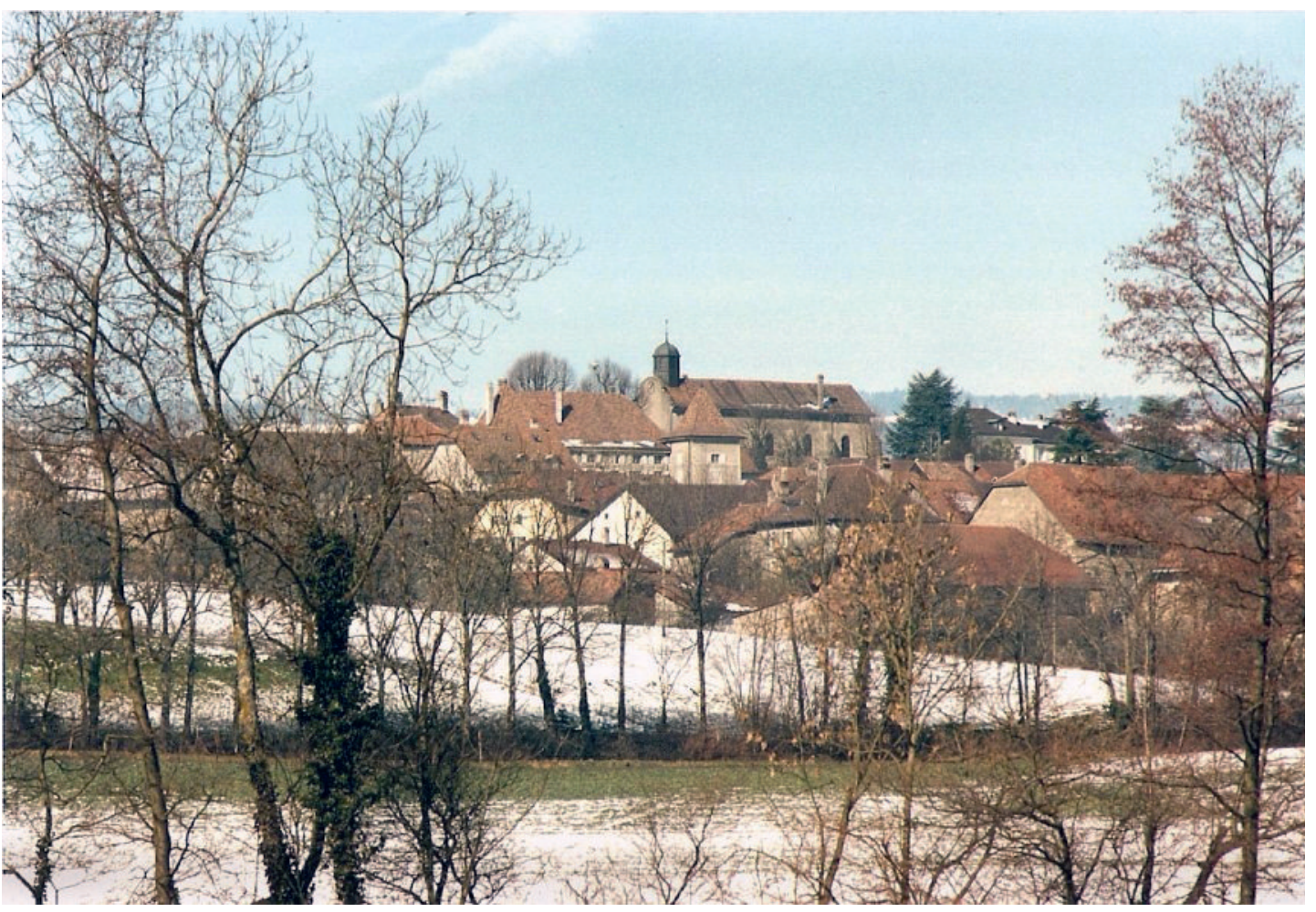


verschiedenen Süchten und Depressionen. Hier kann er ihnen hilfreich zur Seite stehen

Die Notfälle stören, das liegt in ihrer Natur. Aber wie Schwester Julie im Spital Aigle zu sagen pflegte: «Wer das nicht annehmen will, soll diesen Beruf nicht ergreifen und lieber in der Fabrik nebenan Gurken in Blechbüchsen verpacken.» Nun, im Laufe der Jahre habe ich festgestellt, dass die Notfälle in der Tat die Sprechstunde über den Haufen werfen, dass es aber die Patienten nicht unbedingt stört, wenn sie aus dem Wartezimmer nach Hause geschickt werden. Ich

\section{«Auf dem Land ist der Allgemeinpraktiker am rechten Platz, denn da kann er seine erlernten Kenntnisse zur Anwendung bringen.»}

habe einige sogar einsichtig sagen hören: «Ein anderes Mal könnte es uns treffen.» Und wenn der Gipsverband angelegt, die Wundnaht beendet, die Nierensteinkolik bewältigt ist, sage ich mir, dass das Unvorhergesehene auch seine guten Seiten hat. Übrigens treten, wie jeder weiss, gewisse Notsituationen mit Vorliebe zu nächtlicher Stunde auf. Doch auch da: Wenn man nach einer halben Stunde sieht, wie der Pseudokrupp sich löst, wie das Lungenödem auf die Behandlung anspricht, wie die Angehörigen sich beruhigen, empfindet man die Genugtuung, mit einfachen Mitteln dies am Krankenbett erreicht zu haben, ohne gleich die Infrastruktur des Spitals zu beanspruchen.

Es ist eine ständige Herausforderung, das Erlernte in einer realen Situation anzuwenden, nämlich da, wo die Menschen leben und arbeiten, und diese Herausforderung ist anregend für denjenigen, der seinen praktischen und unabhängigen Geist unter Beweis

\section{Begegnung}

Es nachtet schon über der verschneiten Landschaft. Ich kehre von einem letzten Hausbesuch zurück und denke plötzlich an einen Patienten, der heute für ein Rezidiv eines Hirntumors ins CHUV (Waadtländisches Universitätsspital) eingeliefert wurde. Ich denke auch an seinen Vater, der mit ihm allein auf einem einsamen Bauernhof am Fusse der bewaldeten Jurahöhen lebt. In der Zwischenzeit sitzt wohl bereits ein letzter Patient im Wartezimmer. So ist es. Meine Ankunft vor dem grossen Hof wird gleich durch das Bellen des Wachhundes gemeldet. Ich brauche nicht anzuklopfen und warte einen Augenblick vor der Haustüre. Im Hausgang sehe ich die magere Silhouette des früher sicher kräftigen Bauern.

Sein besorgtes Gesicht leuchtet auf, als er mich erkennt. «Sie haben mich nicht erwartet!» «Nicht gerade, aber kommen Sie doch herein!» Wir setzen uns für einen Moment an den grossen Küchentisch unter dem Neonlicht. Im Kochherd wurde soeben ein frisches Stück Holz zugelegt. Das Knistern des Feuers begleitet das Ticken der grossen Wanduhr. Ein einfacher Kalender hängt an der weissen Wand. Die Bibel liegt auf dem Regal. Wir sprechen von den Zeiten, als sich noch mehrmals im Tag die ganze Familie mit den Angestellten um den Tisch versammelte.

Einige Wochen später kommt der Sohn in die Sprechstunde. «lch habe gehört, dass Sie meinen Vater besucht haben! Das hat ihm Freude gemacht. Er hat mir gesagt: «Ein Engel ist gekommen.»» Ein Engel, ich? Warum nicht? ... stellen will. Der Hausbesuch, sei es als Notfall oder Routinearbeit, ist eine Gelegenheit, seine vier Wände zu verlassen und sich mit unvermuteten Realitäten zu konfrontieren. (Es gibt Patienten die ich während vieler Jahre nur in ihrer Wohnung gesehen habe). Der Arzt macht dabei oft Erfahrungen, die zur Verfeinerung seiner ärztlichen Kunst führen und ihn zwingen, die «Guidelines» dem Patienten anzupassen (und nicht umgekehrt ...). Er wird sich bewusst, dass «der Erfolg oder das Misslingen der Medizin oft von kleinen Dingen abhängt», wie es uns einer meiner ehemaligen Professoren lehrte. Patienten lieben diesen Kontakt und verdanken ihn gelegentlich mit sechs Eiern, einem Kürbis oder mit Nüssen. Und meistens geniesse ich die Strecke, erfreue mich der Landschaft, und bei der Fahrt durch die Dörfer kommt mir diese oder jene Person in den Sinn, und ich schicke ihr meine Gedanken.

Und wenn auf der Autobahn wieder ein Stau gemeldet wird, und ich mich allein auf einer schön verschneiten Landstrasse befinde, bin ich froh, hier und nicht anderswo zu sein.

\section{Niederlassung und Anfänge}

Im Dorf Pampigny, am Fuss des Waadtländer Jura, fünfzehn Kilometer von Morges und etwa zwanzig von Lausanne entfernt, eröffneten meine Frau und ich unsere gemeinsame Praxis im Jahr 1978. Damals setzte sich die Bevölkerung jener Gegend fast ausschliesslich aus Landwirten, Holzarbeitern und einigen Handwerkern zusammen. Villen gab es nur wenige.

Fern von allem? So empfinde ich es nicht. Wie viele Dörfer des Landes befindet sich Pampigny nicht allzuweit von der Stadt entfernt, und wir fühlen uns nicht isoliert. Ich kann bestätigen, dass ich mir niemals, nicht einmal im nächtlichen Schneesturm nach einem Konzertbesuch, gesagt habe: «In welches Loch hast du dich vergraben?» Es gibt viel entferntere Gegenden: Vor etlichen Jahren bin ich einem Arzt auf den Shetland-Inseln im Norden Schottlands begegnet. Er war allein für die tausend Einwohner der Insel Yell zuständig, begeistert von seinem Beruf, bedauerte aber doch, niemals eine halbe Stunde mit gutem Gewissen seine Praxis verlassen zu können. Und da eben damals die Verbindungen mit den anderen Inseln schwierig waren, hatten weder er noch die Patienten irgendwelche Wahlmöglichkeiten. Ich meinerseits bin froh, dass die Patienten nicht gezwungen sind, zu mir zu kommen, sondern es tun, weil sie gewillt sind, mit mir zusammenzuarbeiten.

Vieles ist so nah und angenehm. Beginnen wir mit der Praxis, die sich im Erdgeschoss des Wohnhauses befindet. Dadurch wird jeden Tag viel Zeit eingespart; ein Problem für die Pendler, die weit vom Arbeitsplatz wohnen, entfällt. Land und Natur liegen direkt vor der Türe. Was gibt es Wohltuenderes als ein Frühlingsabend, an dem wir von zu Hause weg in den Wald spazieren können? Sonntagabends bewegen sich Autokolonnen in Richtung der Städte. Wir aber 


\section{Begegnung}

Eine Rauchsäule steigt über dem tiefverschneiten Wald auf. Die Kantonalstrasse verlassend, biege ich auf einen Waldweg ab, um unsere Holzfäller zu begrüssen. Sie sind eben daran, eine grosse Weisstanne zu fällen. «Sie muss genau hierhin fallen und sollte sich unter keinen Umständen in dieser Buche verfangen!» Ein letzter Schnitt mit der Motorsäge, und schon fällt der Baum mit einem dumpfen Krachen genau auf die bezeichnete Stelle, gefolgt von einer Pulverschneewolke. Jetzt machen sich die Männer daran, die Äste abzusägen und die Baumrinde zu entfernen. Bald werden sie sich in die Schutzhütte begeben, um ihr Mittagessen einzunehmen, während ich dasselbe mit meiner Familie an der Wärme tun werde.

sind hier, wo wir auch morgen sein werden, denn hier liegt unser Arbeits- und Wohnort. Wir leben in einer angenehmen Dorfgemeinschaft, mit der uns über Jahre entwickelte Beziehungen verbinden. Seit meiner Kindheit und Aufenthalten in den Bergen empfinde ich eine gewisse Faszination für die Welt der Bauern, für Menschen, die im Rhythmus der wisse Zeit, um durch ihre scheinbare Derbheit zu dem vorzudringen, was sie verstecken. Nach dreissig Jahren des Zusammenlebens sind wir uns des Wechsels der Generationen bewusst geworden: Diejenigen, die heute auf den Traktoren sitzen, waren gestern Schulkameraden unserer Kinder.

Stadtbewohner fürchten oft eine gewisse Nähe zur Bevölkerung und ziehen die Anonymität der Stadt vor. Es kommt tatsächlich vor, dass wir auf der Strasse, wie es der Brauch will, ein Gespräch über Banalitäten führen müssen. Die Kehrseite der Medaille ist jedoch die gegenseitige Sorge, welche die Leute in meist zurückhaltender Weise an den Tag legen, die Hilfsbereitschaft und die in aller Einfachheit geleisteten Dienste. Wenn ich bei seltener Gelegenheit ohne Fahrzeug war, hatte ich keine Mühe, mir eines bei einem Nachbarn auszuleihen.

\section{«Dreissig Jahre am gleichen Ort, im selben Beruf, das mag lange erscheinen, aber jeder Tag bringt Neues.»}

Jahreszeiten leben, in Gemeinschaft mit Pflanzen und Tieren. Wie wir sind es unabhängige Menschen, gewohnt, in ihrem Beruf Entscheidungen zu fällen und Risiken einzugehen. Sie legen sich nicht «für nichts und wieder nichts» ins Bett - oft haben sie keine Wahl: Im Stall müssen die Kühe morgens und abends gemolken werden. Zwar braucht es eine ge-
Im Laufe der Jahre hat sich ein Netz von Freunden in der Gegend gebildet (allein schon das Interesse an der Natur ist ein Element der Annäherung und Verbindung). Im Übrigen ist es nicht schwierig, mit entfernter lebenden Freunden Kontakt zu pflegen; sie kommen immer gern zu uns aufs Land zu Besuch. Den Stadtbewohnern fällt die Ruhe auf, die hier zu

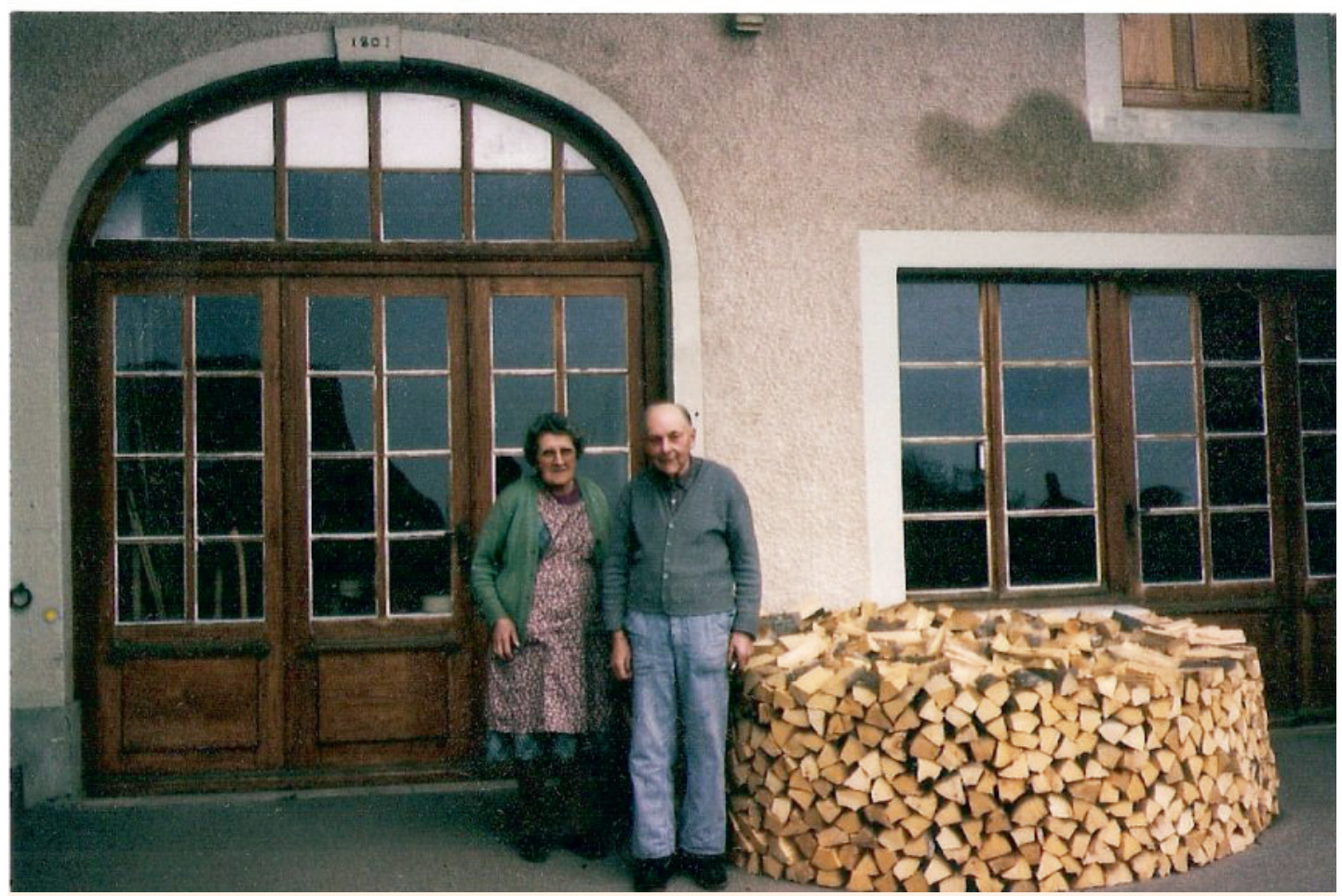

Einige Patienten habe ich in all den Jahren nur zuhause gesehen, niemals in meiner Praxis. 


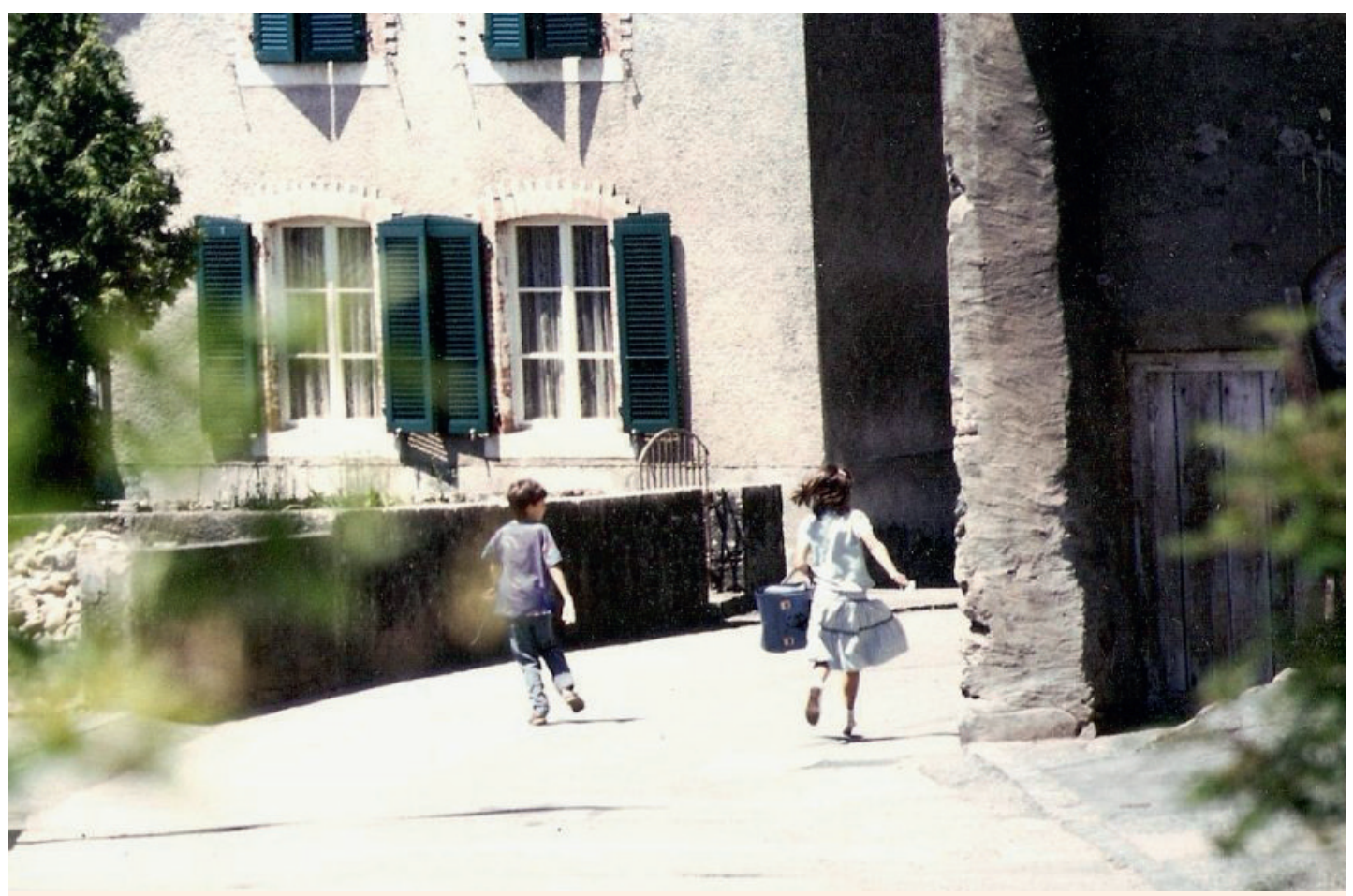

Unsere Kinder auf dem Weg zur Dorfschule.

herrschen scheint, besonders am Abend und während der Nacht. Dem gelegentlichen Strassenlärm folgt der Stundenschlag der Kirchenglocke, das Geläute der weidenden Kühe, das Brummen eines entfernten Flugzeuges oder das Rauschen des Windes im Baumgeäst. Diesbezüglich sind die weite Landschaft und der Raum sicher wichtig. Man erinnere sich an das Experiment der Ethologen, die in einem Aquarium kleine Fische aussetzten und ihren Lebensraum progressiv verkleinerten, somit auch den Abstand zwischen den einzelnen Tieren, wobei letztere immer aufgeregter und aggressiver wurden. Dies ist ein klarer Beweis dafür, dass ein genügender Lebensraum unerlässlich ist. Die Ruhe fördert auch ein angenehmes Arbeitsklima, auch wenn wir uns über die Realitäten keine Illusionen machen. Überlassen wir den Dichtern ihre idealisierten Träumereien. Es besteht kein Grund, dass das Land von Dramen, Leiden und Gewalttätigkeiten verschont bleibt.

\section{Begegnung}

Auf dem Heimweg nach einem Hausbesuch in einer Frühjahrsnacht halte ich meinen Wagen an, lösche die Scheinwerfer und entferne mich zu Fuss. Die Wiesen sind überflutet vom Licht des Vollmondes: Man könnte beinahe ein Reh erkennen, das dem Waldrand entlanggeht. In solch aussergewöhnlichen Momenten lohnt es sich, auf etwas Schlaf zu verzichten. Bald ertönt ein melodischer Ruf am Himmel, zuerst leise, dann immer deutlicher: melancholische, herrliche Flötenstimmen eines Schwarmes von Brachvögeln auf der Migration. Von den Sternen geleitet fliegen die unsichtbaren Stelzenvögel gegen Norden, und ich träume einen Augenblick vom schottischen Heideland, von den Moorlandschaften, vom Wind des Atlantischen Ozeans, der Salz auf die Lippen setzt, und von den grauen Wolken, die einen Regenschauer versprechen ...
Der Landarzt kennt die Kollegen der Nachbardörfer, mit denen er zusammenarbeitet, gut, und auch jeden Spezialisten der nächstliegenden Stadt, dem er seine Patienten konsiliarisch zuweist. Er kennt auch die benachbarten Spitäler, - in meinem Fall diejenigen von Morges und Saint-Loup -, in denen er persönliche Kontakte unterhält, diese der Anonymität der grossen Krankenhäuser vorziehend. Er spricht auch mit den Krankenschwestern und Gehilfen der Spitex-Zentren, deren Arbeit er sehr schätzt. Er kann auch dazu beru-

\section{Ist somit alles perfekt?}

\section{Nein, sicher nicht!}

fen werden, einen Patienten zu pflegen, der die letzten Tage seines Lebens im Kreise seiner Familie verbringen möchte. Dies erfordert oft viel Einsatz und eine grosse Bereitschaft dem Patienten, der Familie sowie allen übrigen Beteiligten gegenüber. Es sind aber auch Momente intensiven Erlebens, für welche die Angehörigen des Patienten oft und lange dankbar sein werden.

Die Praxisassistentin und die Laborantin sind seit mehr als zwanzig Jahren treue und unentbehrliche Stützen meiner Praxis, in der sie die besonders vielfältige Arbeit, welche die Allgemeinmedizin verlangt, mit Kompetenz und Menschlichkeit erledigen. Es kommt kaum mehr als einmal im Winter vor, dass mich die eine oder die andere frühmorgens anruft 
und meldet, sie habe Grippe «- aber es ist schon alles geregelt, meine Kollegin wird mich ersetzen».

Eines Tages erfand jemand in einem entfernten Büro den Ausdruck «Netzwerk». Viele von uns lächelten erstaunt darüber, hatten wir doch diese Neuheit schon längst in natürlicher Weise praktiziert, und vor uns unsere Vorgänger. Niemand hatte das Bedürfnis empfunden, dies mit einem Namen zu versehen.

\section{Die Familie}

Unsere Kinder waren froh, auf dem Lande aufzuwachsen, in die Dorfschule zu gehen und später für die Mittelschule hinunter in die Stadt zu fahren. Auch wenn sie für den Siebenuhrzug früh aufstehen mussten, bleiben die Fahrten verbunden mit Erinnerungen an gute Momente der Kameradschaft mit Kindern aus Nachbardörfern. Beide haben Universitätsstudien absolviert und leben jetzt in Lausanne.

Sowohl für die Kinder wie für die Eltern bietet das Landleben viele Gelegenheiten zu Begegnungen und Freizeitbeschäftigungen: Fussballplatz, Turnhalle, Reithalle, verschiedene Möglichkeiten, sich in künstlerischer Hinsicht zu betätigen, namentlich musizieren, in der Kirchgemeinde und in Jugendorganisationen, beim Singen und vielem anderen. Verschiedene zum Teil informelle Gruppen organisieren Anlässe, Ausstellungen oder Vorträge über dieses oder jenes Thema. Zwei Minuten von unserem Haus entfernt befinden sich die Post, die Bäckerei, das Lebensmittelgeschäft, eine kleine Raiffeisenbank. Kürzlich sagte mir eine junge Mutter, die zwei Jahre zuvor Genf verlassen hatte: «Ich bedauere nur eines: Es nicht früher getan zu haben!»

\section{Und wenn ich es noch einmal tun müsste?}

Wenn Sie mir bis hierher gefolgt sind, erraten Sie meine Antwort. Dreissig Jahre am gleichen Ort, im selben Beruf, das mag lange erscheinen, aber jeder Tag bringt Neues, die Menschen ändern sich, und die Medizin schreitet voran. Viele medizinische Gewissheiten haben anderen Gewissheiten Platz gemacht.

Ist somit alles perfekt? Nein, sicher nicht! Ich kenne auch schwierige Leute, Querulanten und unehrliche, unhöfliche Menschen, sogar einige schlechte Zahler. In Wirklichkeit handelt es sich um eine kleine Minderheit, und meine Beteiligung an einer BalintGruppe hat mir geholfen, diese nicht nur besser anzunehmen und $\mathrm{zu}$ verstehen, sondern auch ihre Bedürfnisse besser zu entziffern. Ich erinnere mich auch - und das berührt mich mehr - an die Krebsfälle, die ich früher hätte entdecken wollen, oder an andere Situationen, in denen ich effizienter hätte handeln sollen. Ohne Zweifel wird ein solches Unbehagen in einer Landgegend stärker empfunden als in der relativen Anonymität der Stadt. Ein erfahrener Diabetologe aus Lausanne hat einmal im Kreise unserer Allgemeinpraktikergruppe gestanden: «Wenn Sie in Pampigny einen schweren Fehler begehen, sind Sie erledigt; wenn es mir passiert, bleibt mir noch der Rest des Kantons.» Ich habe seine Aufrichtigkeit ebensosehr bewundert wie seinen Realismus. Und schliesslich ist es auch normal, dass man an gewissen Tagen weniger Lust zu arbeiten hat als an anderen.

Im Übrigen bin ich mir der administrativen und ökonomischen Schwierigkeiten bewusst, welche die ärztliche Tätigkeit bedrohen, vor allem diejenige der Allgemeinpraktiker. Einen Grossteil der Verantwortung dafür trägt unser ehemaliger Gesundheitsminister Pascal Couchepin, der sich kaum um Gesundheit und Medizin kümmerte, es sei denn in rein wirtschaftlichem Sinne. Um ein günstiges Bild von sich zu geben, hat er von Zeit zu Zeit ein lobendes Wort über die Rolle des Allgemeinarztes im Gesundheitssystem des Landes gesprochen. Gleichzeitig tat er aber alles - und er konnte vieles -, um die Allgemeinpraktiker zu entmutigen. Ich habe einmal in der Presse geschrieben, Pascal Couchepin brauche uns nicht zu lieben, er müsse uns nur arbeiten lassen.

Für die Ärzte und vor allem für die Patienten war zu hoffen, dass sein Nachfolger Didier Burkhalter mehr Menschlichkeit und mehr Verständnis für das reale Leben der Leute zeige und dass sich auch die Verwaltung in dieser Richtung entwickle. Unsere Wünsche wurden aber bisher enttäuscht und unsere Nachfolger werden weiter zu kämpfen haben. Sie werden den Sinn ihrer Arbeit erklären und ein Bild der Medizin zeigen müssen, das etwas attraktiver erscheint als eine Excel-Seite voll Ziffern.

Meine Frau hat vor drei Jahren ihre medizinische Tätigkeit aufgegeben. Eine junge Internistin hat ihre Stelle zu 40\% eingenommen. Unsere Mitarbeit beruht auf einem Vertrag gemäss den Empfehlungen der Waadtländischen Ärztegesellschaft und von Kollegen, die einen ähnlichen Weg gewählt haben. Die Patienten haben unsere Kollegin herzlich empfangen, was bezeugt, falls dies noch nötig wäre, dass die Allgemeinmedizin immer noch einem Bedürfnis entspricht. Eines Tages, so hoffe ich, kommt ein anderer Kollege, so dass ich mich zurückziehen und der jungen Gruppe das Wirkungsfeld überlassen kann.

Es ist am Spätnachmittag eines Sommertages. Einige Kumuluswolken bilden sich über der langen Jurakette. Kinder spielen in der Gasse, drei Sperlinge piepsen am Rand des Daches. Im Dorf riecht es nach frisch eingebrachtem Heu. Ich gehe zur Molkerei, um einige gewohnte Einkäufe zu machen. Als unsere Kinder klein waren, begleitete mich oft das eine oder das andere dabei.

Wie angenehm ist es, auf diese Art nach beendetem Werktag an die Luft zu kommen. Heute war Montag, und trotz vieler Arbeit ist alles gut abgelaufen. Ich tausche einige Worte mit den Bauern, die ihre soeben gemolkene Milch bringen. Auch sie sind am Feierabend nach ihrer Arbeit im Dorf. An manchen Tagen hatten sie mit unerwarteten Ereignissen oder Schwierigkeiten zu tun, an anderen waren sie froh, die Ernte noch rechtzeitig vor dem Gewitter eingebracht zu haben.

Ein alter Bauer, der den Betrieb seinem Sohn übergeben hat, sagte mir eines Tages: «Landwirt ist der schönste Beruf, den es gibt.» Da sagte ich zu mir selbst: Landarzt auch! 